\title{
DNA minor groove hydration probed with 4'-alkylated thymidines
}

\author{
Ilka Detmer, Daniel Summerer and Andreas Marx* \\ Kekulé-Institut für Organische Chemie und Biochemie, Universität Bonn, Gerhard-Domagk-Str. 1, 53121 \\ Bonn, Germany.E-mail: a.marx@uni-bonn.de; Fax: +49228 735388; Tel: +49 228734808
}

Employing modified oligonucleotides that are $4^{\prime}$-alkylated site-specifically we investigated the involvement of DNA minor groove hydration on DNA duplex stability and helix conformation.

Since the first structural investigations of DNA it became evident that hydration of nucleic acids has significant impact on DNA duplex conformation. ${ }^{1}$ One intriguing feature derived from high-resolution structures of DNA oligonucleotides is the presence of intensive hydration throughout the grooves of the DNA double helix. Structures of B-form DNA strongly indicate the presence of an ordered set of water molecules termed 'spine of hydration'. ${ }^{1-3}$ Individual solvent molecules in the hydration spine are coordinated by hydrogen bonding with the nucleobases and the deoxyriboses to bridge both strands. Further recent investigations suggest the coordination of metal ions in the groove as well. ${ }^{3}$ While there is strong evidence for the presence of ordered water molecules in the minor groove, their participation in structural integrity of DNA helix conformations and stability is not well understood and remains to be elucidated.

Here we describe an functional approach to study minor groove hydration through application of oligonucleotides modified site-specifically at the sugar moieties with $4^{\prime}$-alkyl groups (Fig. 1). ${ }^{4}$

Alkyl groups covalently attached at $4^{\prime}$-positions of deoxyribose residues point towards the minor groove of double stranded DNA (Fig. 2). ${ }^{5}$ Thus, they should prevent the formation of an ordered hydrogen spine in the minor groove simply due to steric clash of the ligand with the modification. Varying the bulk of the $4^{\prime}$-modification should allow monitoring the effects of decreased water and ion accessibility of the groove on DNA duplex stability and conformation. It is noteworthy, through deoxyribose modification at the 4'position, that potential effects on hydrogen bonding patterns and stacking properties of the nucleobases are minimized, which significantly distinguish these probes from oligonucleo-
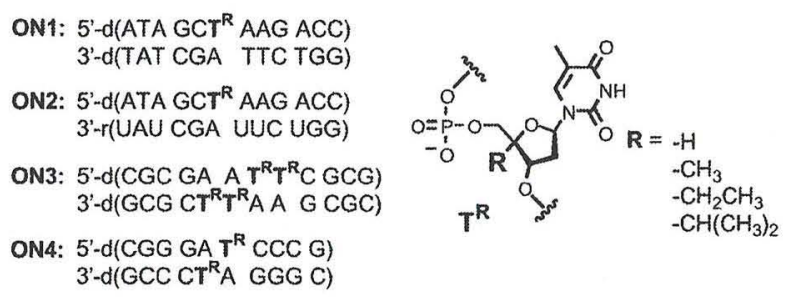

Fig. $1 \uparrow \mathbf{T}^{\mathrm{R}}$ : thymidine residues bearing $4^{\prime}-\mathrm{H}$, methyl, ethyl or isopropyl, respectively. d: 2'-deoxyribose moieties; r: ribose moieties.

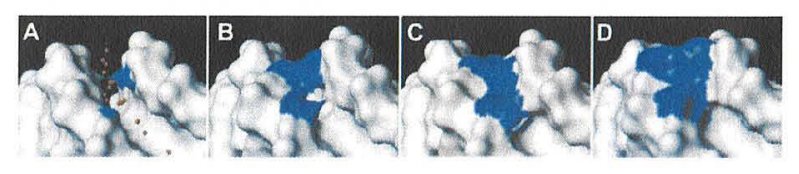

Fig. 2 : tides bearing modified nucleobases, polar $4^{\prime}$-modification, or those that are locked in a particular conformation. ${ }^{4,6-8}$

To subsequently investigate their biophysical properties, we synthesized a series of double stranded oligonucleotides bearing site-specifically either unmodified thymidine or 4'-alkylated thymidine residues by previously described methods (Fig. 1). ${ }^{9}$ Oligonucleotides ON1 and ON2 contain a single modified thymidine residue and their duplex properties were investigated using a complementary DNA and RNA strand, respectively. ON3 $H$ represents the prominent self-complementary Dickerson-Drew DNA dodecamer, which serves as the model for Bform DNA and is studied extensively up to the present day. . $^{1-3}$ Modification of the two central thymidine moieties should result into a subsequent alignment of four successive 4'alkylated thymidines in the central core of the duplex and thus significantly hamper binding of ligands in the central core of the minor groove. Modelling of $4^{\prime}$-alkyl groups into the selected positions of $\mathrm{ON} 3$ suggests that these modifications render the minor groove at the central core increasingly inaccessible to water molecules or ions (Fig. 2). In order to study effects caused by minor groove ligand binding on A-type conformations, selfcomplementary oligonucleotides $\mathrm{ON} 4$ were synthesized, since unmodified ON4 $\mathbf{H}$ is expected to adopt A-type conformation due to its sequence similarity with a previously studied oligonucleotide. ${ }^{10}$

To access the impact of 4 '-alkylation on duplex stability, we performed thermal denaturating studies and measured $T_{\mathrm{m}^{-}}$ values (Table 1). All the measured profiles obtained in thermal denaturation studies showed a single transition. In cases where

Table 1 Thermal denaturating experiments $\left(T_{\mathrm{m}} /{ }^{\circ} \mathrm{C}\right.$ values shown) of oligonucleotides ON1-3 containing either unmodified, 4'-methylated, 4'ethylated or $4^{\prime}$-isopropylated thymidine residues, respectively ${ }^{\prime}$

\begin{tabular}{llll}
\hline Oligonucleotide & $\mathrm{T}^{\mathrm{R}}$ & $T_{\mathrm{m}} /{ }^{\circ} \mathrm{C}$ & $\Delta T_{\mathrm{m}} /{ }^{\circ} \mathrm{C}$ \\
\hline ON1 H & $\mathrm{H}$ & 47.6 & \\
ON1 Me & $\mathrm{Me}$ & 47.1 & -0.5 \\
ON1 Et & $\mathrm{Et}$ & 47.4 & -0.2 \\
ON1 iPr & $i \mathrm{Pr}$ & 46.6 & -1.0 \\
ON2 H & $\mathrm{H}$ & 48.8 & \\
ON2 Me & $\mathrm{Me}$ & 47.8 & -1.0 \\
ON2 Et & $\mathrm{Et}$ & 46.4 & -2.4 \\
ON2 iPr & $i \mathrm{Pr}$ & 45.6 & -3.2 \\
ON3 H & $\mathrm{H}$ & 60.8 & \\
ON3 Me & $\mathrm{Mc}$ & 57.8 & -3.0 \\
ON3 Et & $\mathrm{Et}$ & 53.7 & -7.1 \\
ON3 iPr & $i \mathrm{Pr}$ & 53.8 & -7.0 \\
ON4 H & $\mathrm{H}$ & 53.4 & \\
ON4Me & $\mathrm{Me}$ & 50.5 & -2.9 \\
ON4 Et & $\mathrm{Et}$ & 49.3 & -4.1 \\
ON4 iPr & $i \mathrm{Pr}$ & 48.7 & -4.7
\end{tabular}

${ }^{a}$ Data were obtained from three individual measurements. Melting temperatures $\left(T_{\mathrm{m}} /{ }^{\circ} \mathrm{C}\right.$ values) were obtained from the maximum of first derivative of the melting curve $\left(A_{260} v s\right.$. temperature). Measurements were conducted in buffer comprising $1 \mathrm{M} \mathrm{NaCl}, 20 \mathrm{mM} \mathrm{KH}_{2} \mathrm{PO}_{4}(\mathrm{pH} \mathrm{7.0)}$ ), and contained $600 \mathrm{nM}$ duplex DNA. The mixtures were heated to $95^{\circ} \mathrm{C}$ for 5 min and slowly cooled to room temperature prior to the melting curve measurements. A measurement of the buffer was conducted separately and subtracted from the spectra resulting from the sample. 
only one modified residue per duplex is introduced the data obtained for ON1 exhibit little effects of 4'-methylated or 4'ethylated thymidines on duplex stability compared to the unmodified oligonucleotides. Only a modest decrease of the $T_{\mathrm{m}}{ }^{-}$ value of $1.0{ }^{\circ} \mathrm{C}$ is observed when the bulkiest $4^{\prime}$-modified thymidine $(\mathbf{O N 1} \mathbf{i P r})$ is employed. In cases of binding to RNA (ON2), the measured effects are somehow more pronounced. However, four successive modified 4'-alkyl residues pointing in the minor groove, as present in $\mathbf{O N 3}$, have more significant effects on duplex stability (Table 1). Interestingly, ON3 Et and ON3 iPr exhibit similar thermal stability in the sequence context investigated indicating that potential steric constraints due to the increased bulk are either negligible or compensated by hydrophobic effects. Next, we studied the effects of 4'-alkyl groups on the stability of A-type DNA ON4. Here as well a decrease of duplex stability was observed by introduction of 4 '-alkyl groups into the minor groove (Table 1). Conclusively, our thermal denaturating studies indicate the involvement of minor groove hydration in DNA duplex stability. It is of note that, even after disruption of the hydration spine, oligonucleotides are able to build duplexes.

To explore whether $4^{\prime}$-alkylation has significant impact on overall DNA helix conformation, we examined the circular dichroism (CD) for all native and modified oligonucleotides (Fig. 3). The CD of ON1 H exhibits B-type conformation. The corresponding duplexes bearing $4^{\prime}$-alkyl groups yield superimposable CD spectra indicating little if any dependence of overall helix conformation on the bulk of the $4^{\prime}$-alkyl groups. As expected for a DNA-RNA hybrid, ON2 $\mathbf{H}$ adopts A-type conformation and again the measured $C D$ suggest little perturbation of the overall conformation due to the introduction of single $4^{\prime}$-alkylated thymidine moieties. The Dickerson-Drew DNA dodecamer $\mathbf{O N} \mathbf{3} \mathbf{H}$ displays the conventional B-form. Interestingly, even when bulky $4^{\prime}$-alkyl groups were employed, the duplex adopts B-type conformation with little deviation compared to the native duplex. Nearly overlapping spectra were recorded for modified ON3 indicating little impact of the bulk of the $4^{\prime}$-modifications on overall helix conformation. Investigation of the $\mathrm{CD}$ of $\mathrm{ON} 4$ shows that $4^{\prime}$-alkylation in this sequence context results in no significant alterations of the

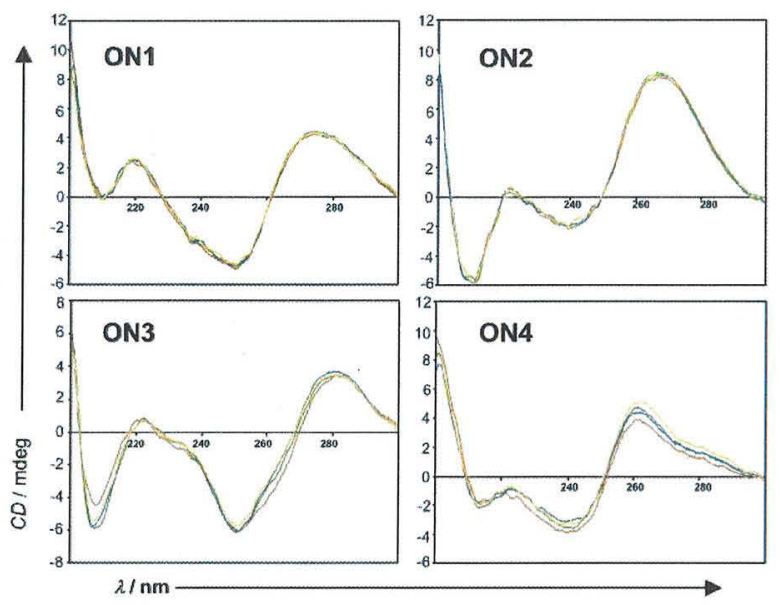

Fig. 3 Circular dichroism spectra of native and $4{ }^{\prime}$-alkylated oligonucleotides. Oligonucleotides investigated are depicted in the figure. Structures and sequences as depicted in Fig. 1. Spectra of native oligonucleotides are displayed in red, 4'-methylated in green, $4^{\prime}$-ethylated in blue, and $4^{\prime}$ isopropylated in orange. $\mathrm{CD}$ spectra were obtained in buffer comprising $1 \mathrm{M}$ $\mathrm{NaCl}, 20 \mathrm{mM} \mathrm{KH}_{2} \mathrm{PO}_{4}$ (pH 7.0), and contained $15 \mu \mathrm{M}$ duplex DNA. The mixtures were heated to $95{ }^{\circ} \mathrm{C}$ for $5 \mathrm{~min}$ and slowly cooled to room temperature prior to measurements. A spectrum of the buffer was measured separately and subtracted from the spectra resulting from the samples. overall helix conformation relative to the native duplex, and all oligonucleotides ON4 form A-type helices. As found before, only minor differences between modified ON4 were observed. In total, the CD data obtained suggest only minor impact of thymidine 4 '-alkylation on the overall conformation of DNA duplexes.

Conclusively, these studies provide functional means for investigation of minor groove hydration or ion binding and its impact on DNA duplex formation and conformation. We found that even in the absence of an ordered hydration spine in the minor groove due to the presence of $4^{\prime}$-alkyl modifications, complementary duplexes are formed, which exhibit little overall helix deviations compared to the unmodified counterparts. However, introduction of several modified residues into oligonucleotide duplexes decreased duplex stability of the respective oligonucleotides.

We thank the DFG for financing this project and M. Famulok for his continuing support.

\section{Notes and references}

$\uparrow$ DNA oligonucleotides synthesised for this study. 4'-alkylated oligonucleotides were synthesized as described. ${ }^{9}$ After cleavage from the solid support all oligonucleotides were purified twice by RP-HPLC (trityl-on and after $5^{\prime}$-deprotection). The integrity of all modified oligonucleotides was confirmed by MALDI-ToF MS.

\# View into the minor groove of ON3. Depicted are solvent accessibility surfaces. 4'-Substituents are shown in blue surfaces. (A) ON3 H, model build on data based on PDB entry 355D. Water molecules present in the structure are depicted as red spheres. (B) ON3 Me; (C) ON3 Et; (D) ON3 iPr.Molecular modelling was conducted as follows: hydrogens were added to the high resolution structure of $\mathrm{ON} 3 \mathrm{H}$ (PDB entry 355D) and minimized as aggregate using the Amber 4.1 force field and the Powell method implemented in SYBYL 6.8 within the Tripos package. Modified duplexes were calculated as follows: alkyl groups were added to above hydrogenminimized structure and were pre-minimized together with the deoxyribose unit as monomers using the MMFF94 force field and the Powell method. The respective duplexes were subsequently minimized with the Amber 4.1 force field and the Powell method. Instead of explicit solvent molecules, a distant dependent dielectric constant of $4 r$ was used. Surfaces correspond to solvent accessibility using a probe radius of $1.4 \AA$

1 (a) R. E. Dickerson, Methods Enzymol., 1992, 211, 67; (b) S. Neidle, Nat. Struct. Biol., 1998, 5, 754; (c) H. M. Berman and B. Schneider in Oxford Handbook of Nucleic Acid Structure, ed. S. Neidle, Oxford University Press, New York, 1999295.

2 R. Wing, H. Drew, T. Takano, C. Broka, S. Takana, K. Itakura and R. E. Dickerson, Nature, 1980, 287, 755 .

3 (a) Recent examples: X. Shui, L. McFail-Isom, G. G. Hu and L. D. Williams, Biochemistry, 1998, 37, 8341; (b) M. Soler-López, L Malinina, J. Lui, T. Huynh-Dinh and J. A. Subirana, J. Biol. Chem., 1999, 274, 23683; (c) V. Tereshko, G. Minasov and M. Egli, J. Am. Chem. Soc., 1999, 121, 470; (d) V. Tereshko, G. Minasov and M. Egli, J. Am. Chem. Soc., 1999, 121, 3590.

4 (a) For functional approaches using nucleobase analogues, see: T. Lan and L. W. McLaughlin, J. Am. Chem. Soc., 2000, 122, 6512; (b) T. Lan and L. W. McLaughlin, Biochemistry, 2001, 40, 968.

5 W. Saenger, Principles of Nucleic Acid Structure, ed. C. Cantor, Springer, New York, 1993; Oxford Handbook of Nucleic Acid Structure, ed. S. Neidle, Oxford University Press, New York, 1999.

6 Recently, a similar approach was suggested using $5^{\prime}$-alkylated nucleotides: H. Trafelet, E. Stulz and C. Leumann, Helv. Chim. Acta, 2001, 84, 87.

7 (a) G. Wang and W. F. Seifert, Tetrahedron Lett., 1996, 37, 6515; (b) M. Kanazaki, Y. Ueno, S. Shuto and A. Matsuda, J. Am. Chem. Soc., 2000 , 122, 2422.

8 (a) Recent reviews: J. Wengel, Acc. Chem. Res., 1999, 32, 301; (b) L. Kværnø and J. Wengel, Chem. Commun., 2001, 1419.

9 D. Summerer and A. Marx, Angew. Chem., Int. Ed., 2001, 40, 3693; D. Summerer and A. Marx, J. Am. Chem. Soc., 2002, 124, 911; M. Strerath, D. Summerer and A. Marx, ChemBioChem, 2002, 3, 578.

10 H. Laube, R. Frank, H. Blöcker and U. Heinemann, Nucleic Acids Res., 1988, 16, 7799. 